\title{
Eclipsing Binaries in the LMC: a Wealth of Data for Astrophysical Tests
}

\author{
A. Derekas, L. L. Kiss and T. R. Bedding \\ School of Physics, University of Sydney, NSW 2006, Australia \\ email: derekas@physics.usyd.edu.au
}

\begin{abstract}
.
We have analysed publicly available MACHO observations of 6833 variable stars in the Large Magellanic Cloud, classified as eclipsing binaries. After finding that a significant fraction of the sample was misclassified, we redetermined periods and variability class for all stars, producing a clean sample of 3031 eclipsing binaries. We have investigated their distribution in the periodcolor-luminosity space, which was used, for example, to assign a foreground probability to every object and establish new period-luminosity relations to selected types of eclipsing stars. We found that the orbital period distribution of LMC binaries is very similar to those of the SMC and the Milky Way. We have also determined the rate of period change for every star using the $O-C$ method, discovering about 40 eclipsing binaries with apsidal motion, 45 systems with cyclic period changes and about 80 stars with parabolic $O-C$ diagrams. In a few objects we discovered gradual amplitude variation, which can be explained by changes in the orbital inclination caused by a perturbing third body in the system.
\end{abstract}

Keywords. binaries: eclipsing, galaxies: individual (Large Magellanic Cloud)

\section{Introduction}

The last decade witnessed the birth of a new research field, the large-scale study of variable stars in external galaxies. This has first been made possible by the huge databases of microlensing observations of the Magellanic Clouds, like the MACHO, OGLE and EROS projects (see Szabados \& Kurtz 2000 for reviews). These programs (beyond their primary purpose) resulted in the discovery of thousands of new eclipsing binaries with an unprecedented homogeneous coverage of their light curves opening a new avenue of the binary star research. Here we present the first results of an analysis of the publicly available MACHO light curves. The main aim of the project is to measure period changes and discover eclipsing binaries with pulsating components.

\section{General properties of the sample}

We have analysed MACHO lights curves for 6833 stars that were originally classified as eclipsing binaries. After re-determining the period and classifying the stars based on their light curve shapes, only 3031 stars remained as genuine eclipsing or ellipsoidal variables (the rest being Cepheids, RR Lyraes and other non-eclipsing variable stars). The period distribution of this binary sample is bimodal, with the strongest peak between 1 and 2 days. Roughly $20 \%$ of stars have periods longer than 10 days; many of them show W UMa-like light curve shapes, suggesting ellipsoidal variability of giant componens.

We classified the binary sample using Fourier decomposition of the phase diagrams. Two coefficients, $\mathrm{a}_{2}$ and $\mathrm{a}_{4}$, of the cosine decomposition $\sum_{\mathrm{i}=1}^{4} \mathrm{a}_{\mathrm{i}} \cos (2 \pi \mathrm{i} \varphi)$ allow a welldefined distinction between detached, semi-detached and contact binaries (Pojmański 2002). The results show that the sample is dominated by bright main-sequence detached 


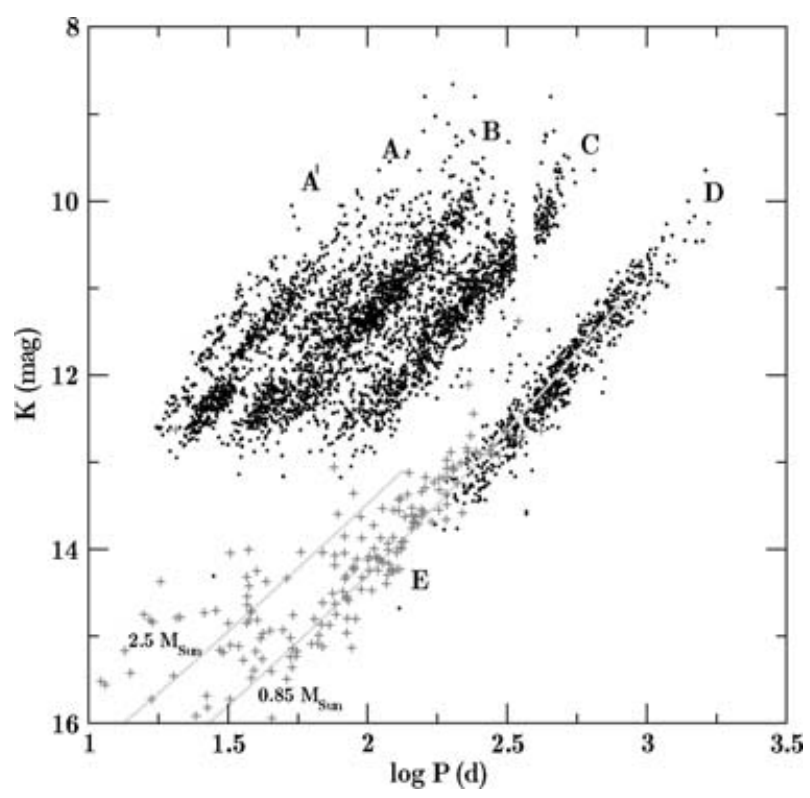

Figure 1. P-L relations of ellipsoidal variables (red pluses) red giant pulsators (black dots).

The two lines show a simple model using evolutionary tracks and Roche-geometry.

(50\%) and semi-detached (30\%) binaries. Contact systems comprise $20 \%$ of the sample; the short-period systems are all foreground objects in the Milky Way, while longer periods belong to red giant binaries.

We used the Color-Magnitude Diagram (CMD) to clean the sample of the foreground objects. For this we took evolutionary models of Castellani et al. (2003) and calculated the locations of certain minimal orbital period values (where two identical model stars are in contact). The cleaned sample contains about 2800 LMC binaries.

Detached and semi-detached binaries are spread uniformly in the period $-K$ magnitude plane, while there is a well-defined sequence for the contact systems. We found that the widely accepted sequence of eclipsing binaries between Seqs. C and D, known as Seq. E, does not exist. The correct position for Seq. E is at periods a factor of two greater. A simple Roche-model describes Seq. E very well. Although Seq. E seems to merge into Seq. D of the Long Secondary Periods (Wood et al. 2004), the two groups are significantly different in their color and amplitude properties (Derekas et al. 2006).

\section{Period changes and secular amplitude variations}

From the 8 years of MACHO observations we measured period changes using the $O-C$ method applied to seasonal subsets of the data. We found about 80 parabolic and 45 cyclic period changes, the rest showing linear $O-C$ diagrams. A significant fraction of the former two groups are candidates for light-time effect in hierarchic triple systems. One example is shown in the top panels of Figure 2. In about 40 eccentric binaries we measured different $O-C$ variations for the primary and the secondary minima, which indicates apsidal motion (bottom panels in Figure 2). With this we double the number of known binaries with apsidal motion in the LMC (Michalska \& Pigulski 2005).

In a few objects we discovered gradual amplitude variation, which can be explained by rapid variations in the orbital geometry, most likely in inclination. A third body in the system can perturb the eclipsing pair in such a way that the eclipse depth, as a sensitive 

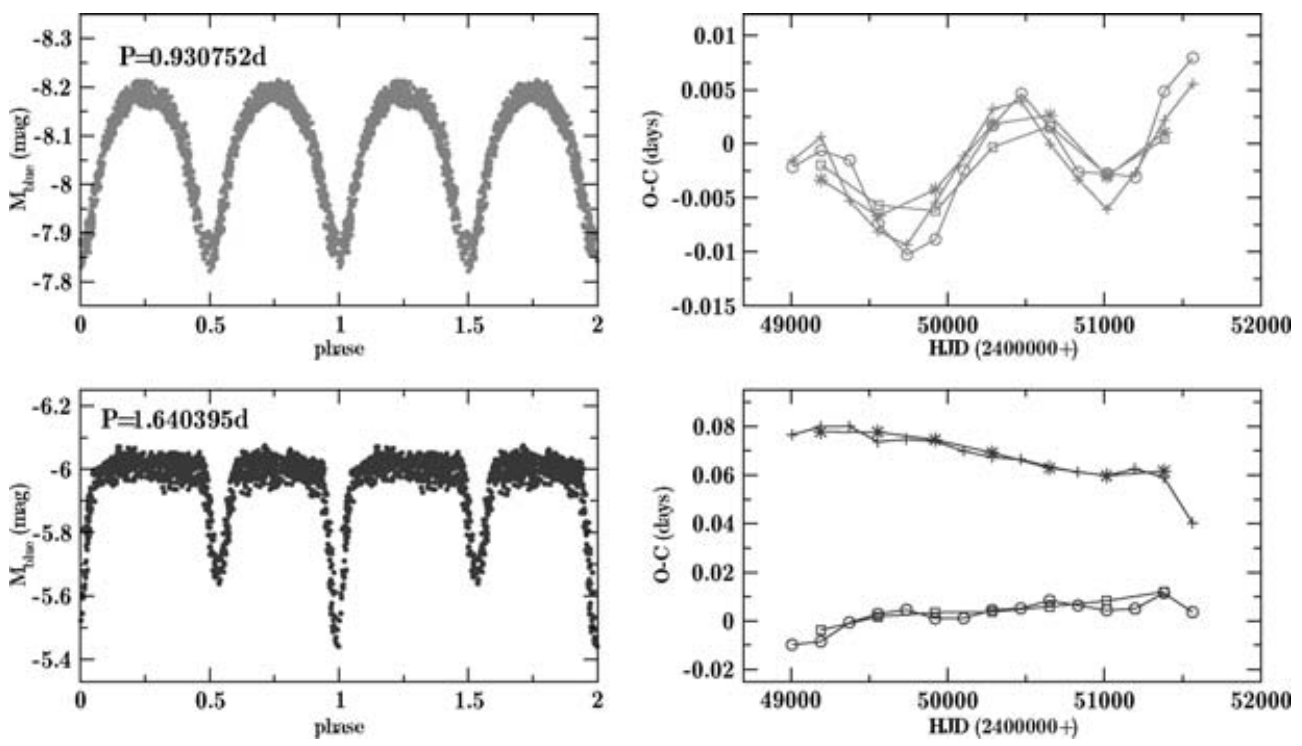

Figure 2. Examples for cyclic $O-C$ diagram and apsidal motion.
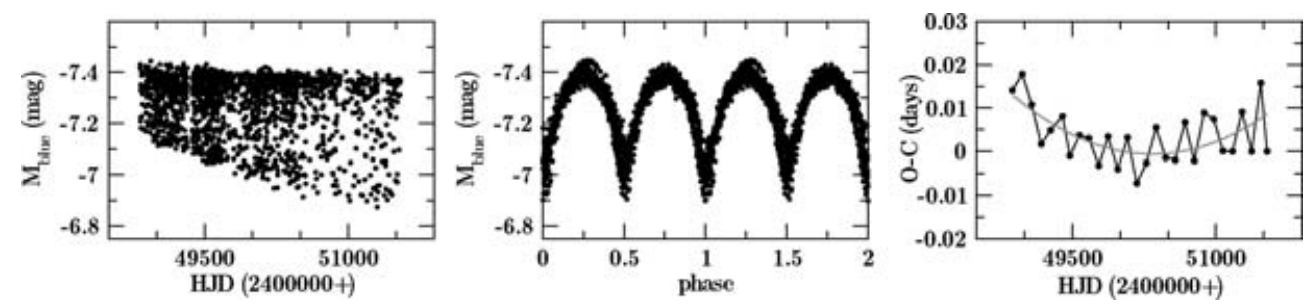

Figure 3. An example for changing minimum depth.

indicator of the inclination variations, follows these perturbations. In Figure 3 we present an example $\left(\mathrm{P}_{\mathrm{ecl}}=0.77 \mathrm{~d}\right)$, for which the large scatter in the $O-C$ diagram may suggest an orbital period of $\leqslant 100 \mathrm{~d}$ for the hypothetical third companion.

\section{Acknowledgements}

This work has been supported by the Australian Research Council. LLK is supported by a University of Sydney Postdoctoral Research Fellowship. AD is supported by an Australian Postgraduate Research Award. This paper utilizes public domain data obtained by the MACHO Project, jointly funded by the US Department of Energy through the University of California, Lawrence Livermore National Laboratory under contract No. W-7405-Eng-48, by the National Science Foundation through the Center for Particle Astrophysics of the University of California under cooperative agreement AST-8809616, and by the Mount Stromlo and Siding Spring Observatory, part of the Australian National University.

\section{References}

Castellani, V., et al., 2003, A\& A, 404, 645

Derekas, A. et al., 2006, ApJL, in press (astro-ph/0608618)

Michalska, G. \& Pigulski, A., 2005, A\& $A, 434,89$

Pojmański, G., 2002, Acta Astron., 52, 397

Szabados, L. \& Kurtz, D.W., (Eds.), 2000, ASP Conf. Series, Vol. 203

Wood P.R., Olivier, E.A., \& Kawaler, S.D., 2004, ApJ, 604, 800 